\title{
MULTIPLICITY OF BOARDMAN STRATA AND DEFORMATIONS OF MAP GERMS
}

\author{
by J. J. NUÑO BALLESTEROS* and M. J. SAIA†
}

(Received 9 May, 1996)

\begin{abstract}
We define algebraically for each map germ $f: K^{n}, 0 \rightarrow K^{p}, 0$ and for each Boardman symbol $\mathbf{i}=\left(i_{1}, \ldots, i_{k}\right)$ a number $c_{\mathbf{i}}(f)$ which is $\mathscr{A}$-invariant. If $f$ is finitely determined, this number is the generalization of the Milnor number of $f$ when $p=1$, the number of cusps of $f$ when $n=p=2$, or the number of cross caps when $n=2, p=3$. We study some properties of this number and prove that, in some particular cases, this number can be interpreted geometrically as the number of $\Sigma^{\mathbf{i}}$ points that appear in a generic deformation of $f$. In the last part, we compute this number in the case that the map germ is a projection and give some applications to catastrophe map germs.
\end{abstract}

1. Introduction. The Milnor number of an analytic function germ $f: \mathbb{C}^{\prime \prime}, 0 \rightarrow \mathbb{C}$ which has isolated singularity at zero is defined as

$$
\mu(f)=\operatorname{dim}_{\mathbb{C}} \frac{\mathscr{E}_{n}}{J_{f}}
$$

where $\mathscr{E}_{n}$ is the ring of germs $\mathbb{C}^{\prime \prime}, 0 \rightarrow \mathbb{C}$ and $J_{f}$ is the jacobian ideal generated by the partial derivatives $\partial f / \partial x_{i}$, for $i=1, \ldots, n$. It is well known that this number can be interpreted geometrically as the number of Morse points (or $\Sigma^{n, 0}$ points if we use the Thom-Boardman singularities notation) that appear in a stable deformation of $f$.

Analogously, if we consider a finitely determined map germ $f: \mathbb{C}^{2}, 0 \rightarrow \mathbb{C}^{2}, 0$, we can define the number

$$
c(f)=\operatorname{dim}_{\mathbb{C}} \frac{\mathscr{E}_{2}}{\left\langle J, p_{x} J_{y}-p_{y} J_{x}, q_{x} J_{y}-q_{y} J_{x}\right\rangle},
$$

where $f=(p, q), J$ is the Jacobian determinant and subscripts indicate partial derivatives. Then, according to [2] or [4], we have that this number is the number of cusps (i.e., $\Sigma^{1.1,0}$ points) that appear in a stable deformation of $f$.

Finally, a similar result can be found in [7] for a finitely determined map germ $f: \mathbb{C}^{2}, 0 \rightarrow \mathbb{C}^{3}, 0$. The number $c(f)$ is defined as

$$
c(f)=\operatorname{dim}_{\mathbb{C}} \frac{\mathscr{E}_{2}}{\left\langle J_{1}, J_{2}, J_{3}\right\rangle},
$$

where $J_{1}, J_{2}, J_{3}$ are the three 2-minors of the jacobian matrix of $f$. In this case, the number $c(f)$ is the number of cross caps (i.e., $\Sigma^{1.0}$ points) that appear in a stable deformation of $f$.

Here, we generalize the three constructions by defining a number $c_{\mathrm{i}}(f)$, for each Boardman symbol $\mathbf{i}=\left(i_{1}, \ldots, i_{k}\right)$ and for each map germ $f: K^{\prime \prime}, 0 \rightarrow K^{\prime \prime}, 0$ (real analytic if $K=\mathbb{R}$ or analytic if $K=\mathbb{C}$ ). We prove that this number is $\mathscr{A}$-invariant and study some properties.

* Work partially supported by DGICYT Grant PB93-0707.

† Work partially supported by FAPESP.

Glasgow Math. J. 40 (1998) 21-32. 
The main part of this paper is dedicated to answer the following question: let $f: \mathbb{C}^{n}, 0 \rightarrow \mathbb{C}^{p}, 0$ be a finitely determined map germ and $\mathbf{i}$ a Boardman symbol such that $\Sigma^{\mathbf{i}}$ has codimension $n$ in the corresponding jet space $J^{k}(n, p)$, when is $c_{\mathrm{i}}(f)$ equal to the number of $\Sigma^{\mathbf{i}}$ points that appear in a generic deformation of $f$ ? Here, generic means generic in the sense of Thom-Boardman (that is, the jet extension of the map germ is transversal to all the Boardman submanifolds). We prefer the concept of generic deformation instead of stable deformation because a stable deformation does not always exist, unless you are in the "nice dimensions" of Mather [5]. Our partial answer to this question is that the result is true in three situations (see Theorem 4.4), namely: 1) $i$ has length 1,2$) f$ is a singularity of type $\Sigma^{\mathbf{i}}$, or 3$) f$ has rank $n-1$ and $\mathbf{i}=(1, \ldots, 1)$. We also show in Example 4.5 that the result is not true in general and give and example of a map germ $f: \mathbb{C}^{3}, 0 \rightarrow \mathbb{C}^{3}, 0$ so that $c_{1.1 .1}(f)$ is not equal to the number of $\Sigma^{1.1 .1}$ points that appear in a generic deformation of $f$.

In the last section, we study this number in the case that the map germ is a projection $\pi: X, x \rightarrow K^{\prime \prime}, 0$, where $(X, x)$ is the set germ of zeros of a map germ $g: K^{N}, 0 \rightarrow K^{p}, 0$, which has 0 as a regular value. We compute the number $c_{\mathrm{i}}(\pi)$ in terms of the map germ $g$ and prove that it depends only on the partial derivatives of $g$ with respect to the coordinates which are not projected by $\pi$. In particular, this result has some applications to catastrophe map germs

AcKNowledgment. The main part of this work has been done during the authors' stay at the University of Liverpool. The authors would like to thank members of Singularity Seminar in the Department of Pure Mathematics for their hospitality, and specially J. W. Bruce and F. Tari for helpful comments. Thanks are also due to D. Mond, who pointed out a mistake in previous version of this paper.

2. Definition of the invariant. Let $f: K^{n}, 0 \rightarrow K^{p}, 0$ be a map germ $\left(C^{\infty}\right.$ or smooth if $K=\mathbb{R}$ or analytic if $K=\mathbb{C}$ ). We shall denote by $\mathscr{E}_{n}$ the ring of germs $K^{n}, 0 \rightarrow K$. We recall here a construction due to Morin (see [9]).

Definition 2.1. Let $f: K^{n}, 0 \rightarrow K^{p}$, be a map germ and $I \subset \mathscr{E}_{n}$ an ideal generated by elements $g_{1}, \ldots, g_{r} \in I$. Then for each $m=1, \ldots, n$ we define the jacobian extension of rank $m$ of $(f, l)$ as

$$
\Delta_{m}(f, I)=I+I^{\prime},
$$

where $I^{\prime}$ is the ideal generated by the minors of order $m$ of the jacobian matrix of $\left(f_{1}, \ldots, f_{p}, g_{1}, \ldots, g_{r}\right)$.

When $f=0$, we put $\Delta_{m}(0, I)=\Delta_{m}(I)$, and thus this construction coincides with the jacobian extension defined by other authors.

Lemma 2.2. Suppose that $f: K^{n}, 0 \rightarrow K^{p}, 0$ is a map germ, $l \subset \mathscr{C}_{n}$ an ideal, and $h: K^{n}$, $0 \rightarrow K^{n}, 0, k: K^{p}, 0 \rightarrow K^{p}, 0$ are diffeomorphism germs. Then we have

(i) the ideal $\Delta_{m}(f, I)$ does not depend on the generators chosen for the ideal $I$;

(ii) $\Delta_{m}\left(f \circ h, h^{*} I\right)=h^{*}\left(\Delta_{m}(f, I)\right)$, where $h^{*}: \mathscr{E}_{n} \rightarrow \mathscr{E}_{n}$ is the induced isomorphism of $K$-algebras;

(iii) $\Delta_{m}(k \circ f, I)=\Delta_{m}(f, I)$;

(iv) $\Delta_{m}(f, I)+I_{f}=\Delta_{m}\left(I+I_{f}\right)$, where $I_{f}=\left\langle f_{1}, \ldots, f_{p}\right\rangle$. 
Proof. We prove the second property, which is perhaps the least obvious. Suppose that the ideal $I$ is generated by $g_{1}, \ldots, g_{r}$. Then $h^{*} I$ is generated by $h^{*} g_{1}, \ldots, h^{*} g_{r}$. On the other hand, the chain rule gives that

$$
\frac{\partial f_{i} \circ h}{\partial x_{j}}=\sum_{k=1}^{n}\left(\frac{\partial f_{i}}{\partial x_{k}} \circ h\right) \frac{\partial h_{k}}{\partial x_{j}}=\sum_{k=1}^{n} h^{*}\left(\frac{\partial f_{i}}{\partial x_{k}}\right) \frac{\partial h_{k}}{\partial x_{j}} .
$$

This implies that every $m$-minor $d$ of the jacobian matrix of $\left(f \circ h, h^{*} g\right)$ can be written as a linear combination $d=\sum a_{i} h * d_{i}$, where $a_{i} \in \mathscr{E}_{n}$ and $d_{i}$ are $m$-minors of the jacobian matrix of $(f, g)$. Therefore $\Delta_{m}\left(f \circ h, h^{*} l\right) \subset h^{*}\left(\Delta_{m}(f, I)\right)$.

The opposite inclusion follows by applying the same argument to the map germ $f^{\prime}=f \circ h$, the ideal $J=h^{*} I$ and the diffeomorphism germ $h$.

It will also be useful in the following definitions to take the convention that $\Delta_{n+1}(f, l)=l$ for any map germ $f$ and ideal $I$.

Definition 2.3. Let $f: K^{n}, 0 \rightarrow K^{p}, 0$ be a map germ and $\mathbf{i}=\left(i_{1}, \ldots, i_{k}\right)$ a Boardman symbol (i.e., $n \geq i_{1} \geq \ldots \geq i_{k} \geq 0$ ). Then we define inductively the iterated jacobian extension of $f$ by

$$
J_{\mathbf{i}}(f)= \begin{cases}\Delta_{n-i_{1}+1}(f,\{0\}), & \text { if } k=1 \\ \Delta_{n-i_{k}+1}\left(f, J_{i_{1} \ldots, i_{k-1}}(f)\right), & \text { if } k>1 .\end{cases}
$$

Moreover, we define the number $c_{\mathrm{i}}(f)$ by:

$$
c_{\mathrm{i}}(f)=\operatorname{dim}_{K} \frac{\mathscr{E}_{n}}{J_{\mathbf{i}}(f)} .
$$

EXAMPLES. When $p=1$ and $\mathbf{i}=n$, we have that $J_{n}(f)$ is the ideal $\left\langle\partial f / \partial x_{1}, \ldots, \partial f / \partial x_{n}\right\rangle$. Thus, if $f$ has isolated singularity at zero, $c_{n}(f)$ is just the Milnor number of $f$. In the complex case this number can be interpreted as the number of Morse points (i.e., $\Sigma^{\prime \prime}$ points) that appear in a stable deformation of $f$. In the real case, $c_{n}(f)$ would just be the maximum of this number.

When $p=n=2$ and $\mathbf{i}=(1,1)$, if the map germ is given by $f=(p, q)$, the ideal $J_{1,1}(f)$ is generated by $J, p_{x} J_{y}-p_{y} J_{x}, q_{x} J_{y}-q_{y} J_{x}$, where $J$ is the Jacobian determinant and subscripts indicate partial derivatives. According to $[2,3]$ and again in the complex case, if $f$ is finitely determined, $c_{1.1}(f)$ is the number of cusps (i.e., $\Sigma^{1,1}$ points) which appear in a stable deformation of $f$.

And finally, the same happens for the case $p=2, n=3$ and $\mathbf{i}=1$ with the number of cross caps (see [7] for more details).

Notation. Suppose that we select a fixed set of coordinates $x_{i_{1}}, \ldots, x_{i_{r}}$ of $K^{\prime \prime}$. We can construct the jacobian extension $\Delta_{m}(f, I)$ by looking only at the partial derivatives with respect to these coordinates. We shall denote this by putting $\Delta_{m}\left(f, I ; x_{i_{1}}, \ldots, x_{i_{r}}\right)$. Then we use $J_{\mathbf{i}}\left(f ; x_{i_{1}}, \ldots, x_{i_{r}}\right)$ for the corresponding iterated jacobian extension and $c_{i}\left(f ; x_{i}, \ldots, x_{i_{r}}\right)$ for the number.

Proposition 2.4. The number $c_{\mathrm{i}}(f)$ is $\mathscr{A}$-invariant.

Proof. Suppose that $f, g$ are $\mathscr{A}$-equivalent. Then $g=k \circ f \circ h$ for some 
diffeomorphism germs $h, k$. By properties 2 and 3 of Lemma 2.2, we have that $J_{\mathbf{i}}(g)=h^{*} J_{\mathbf{i}}(f)$. Thus, $J_{\mathbf{i}}(f), J_{\mathbf{i}}(g)$ are induced isomorphic and $c_{\mathbf{i}}(f)=c_{\mathbf{i}}(g)$.

Remark. Although the theory of Boardman symbols was introduced in the context of $\mathscr{K}$-equivalence, the number $c_{\mathbf{i}}(f)$ is not $\mathscr{K}$-invariant. For instance, consider the map germs $f(x, y)=\left(x, x y+y^{3}\right), g(x, y)=\left(x, y^{3}\right)$, which are $\mathscr{K}$-equivalent. However, $c_{1,1}(f)=$ 1 and $c_{1,1}(g)=\infty$.

Suppose now that $f: K^{n}, 0 \rightarrow K^{p}, 0$ is a map germ of rank $r$. We know that after a coordinate change in the source, $f$ can be written as an unfolding of a map germ $K^{n-r}$, $0 \rightarrow K^{p-r}$, 0 . That is, we can put $f(u, x)=(u, g(u, x))$, where $u, x$ denote coordinates in $K^{r}, K^{n-r}$ respectively, and $g: K^{n}, 0 \rightarrow K^{p-r}, 0$ is a map germ. The next proposition says that in this case, the number $c_{i}(f)$ is easier to compute.

Proposition 2.5. Suppose that $f: K^{n}, 0 \rightarrow K^{p}, 0$ is a map germ given by $f(u, x)=$ $(u, g(u, x))$, for $u \in K^{r}, x \in K^{n-r}$ and let $\mathbf{i}=\left(i_{1}, \ldots, i_{k}\right)$. Then

$$
c_{\mathbf{i}}(f)= \begin{cases}0, & \text { if } i_{1}>n-r \\ c_{\mathbf{i}}(g ; x), & \text { if } i_{1} \leq n-r .\end{cases}
$$

Proof. The jacobian matrix of $f$ has the form $\left(\begin{array}{ll}I_{r} & A \\ 0 & B\end{array}\right)$, where $I_{r}$ is the identity matrix of order $r, A=\left(\partial g_{i} / \partial u_{j}\right)$ is the jacobian matrix of $g$ with respect to the coordinates $u_{j}$ and $B=\left(\partial g_{i} / \partial x_{j}\right)$ is the jacobian matrix with respect to the coordinates $x_{j}$.

In the case that $i_{1}>n-r$, we have $n-i_{1}+1 \leq r$. This gives that there is a minor of order $n-i_{1}+1$ which is equal to 1 . Thus $J_{i_{1}}(f)=\mathscr{C}_{n}$ and $c_{\mathrm{i}}(f)=0$.

Otherwise, $n-i_{1}+1>r$ and every $\left(n-i_{1}+1\right)$-minor coincides with a minor of $B$ of order $\geq n-r-i_{1}+1$. Reciprocally, every $\left(n-r-i_{1}+1\right)$-minor of $B$ can be seen as a $\left(n-i_{1}+1\right)$-minor of the whole matrix. This proves that $J_{i_{1}}(f)=J_{i_{1}}(g ; x)$.

Now we proceed by induction and applying a similar argument in each step, we get $J_{\mathbf{i}}(f)=J_{\mathbf{i}}(g ; x)$, which concludes the proof.

Corollary 2.6. Suppose that $f: K^{n}, 0 \rightarrow K^{p}, 0$ is a map germ of rank $n-1$ given by $f(u, x)=(u, g(u, x))$, for $u \in K^{n-1}, x \in K$ and let $\mathbf{i}=\left(i_{1}, \ldots, i_{k}\right)$. Then

$$
c_{\mathbf{i}}(f)= \begin{cases}0, & \text { if } i_{1}>1 \\ \operatorname{dim}_{K} \frac{\mathscr{E}_{n}}{\left\langle\frac{\partial g_{1}}{\partial x}, \ldots, \frac{\partial g_{p-n+1}}{\partial x}, \ldots, \frac{\partial^{s} g_{1}}{\partial x^{s}}, \ldots, \frac{\partial^{s} g_{p-n+1}}{\partial x^{s}}\right\rangle}, & \text { if } i_{1}=1,\end{cases}
$$

being the number $s$ in the second case equal to the number of indices $i$, which are not zero.

3. Relation with the Thom-Boardman singularities. We recall the definition of the Thom-Boardman singularities, taking into account our notation. We say that a map germ $f: K^{\prime \prime}, 0 \rightarrow K^{p}, 0$ is a singularity of type $\Sigma^{\mathbf{i}}$, for a Boardman symbol $\mathbf{i}=\left(i_{1}, \ldots, i_{k}\right)$, when

(i) the rank of $f$ is $n-i_{1}$; 
(ii) for all $s=2, \ldots, k$, the rank of $(f, g)$ is $n-i_{s}$, being $g=\left(g_{1}, \ldots, g_{r}\right)$ and $g_{1}, \ldots, g_{r}$ generators of $J_{i_{1}, \ldots, i_{1-1}}(f)$.

We denote by $\Sigma^{\mathrm{i}}(f)$ the set germ of points $x$, such that the germ of $f$ at $x$ is a singularity of type $\Sigma^{\mathbf{i}}$. Remember also that this set germ $\Sigma^{\mathbf{i}}(f)$ can be written as:

$$
\Sigma^{\mathbf{i}}(f)=\left(j^{k} f\right)^{-1}\left(\Sigma^{\mathbf{i}}\right),
$$

where $\Sigma^{\mathbf{i}}$ is the corresponding Boardman submanifold defined in the jet space $J^{k}(n, p)$.

To see the relationship between these sets and the ideals $J_{\mathbf{i}}(f)$ defined in the above section, we need to introduce some notation used by Morin in [9].

We define the lexicographic order, $\leq$, in the set of Boardman symbols, that saying that $\mathbf{i} \leq \mathbf{j}$ if writing $\mathbf{i}=\left(i_{1}, \ldots, i_{k}\right)$ and $\mathbf{j}=\left(j_{1}, \ldots, j_{l}\right)$, we have that either $\mathbf{i}=\mathbf{j}$ or $i_{r_{11}}<j_{r_{1}}$, where $r_{0}=\min \left\{r: i_{r} \neq j_{r}\right\}$.

The length, $|\mathbf{i}|$, of a Boardman symbol $\mathbf{i}=\left(i_{1}, \ldots, i_{k}\right)$ is defined as the last $r$ such that $i_{r}>0$.

Given a Boardman symbol $\mathbf{i}=\left(i_{1}, \ldots, i_{k}\right)$, we define its successor as the symbol $\mathbf{i}^{\prime}$ which is the following symbol for the lexicographic order among the symbols $\mathbf{j}$ such that $|\mathbf{j}| \leq|\mathbf{i}|$. That is, $\mathbf{i}^{\prime}=\left(i_{1}, \ldots, i_{r}, i_{r+1}+1\right)$, provided that $i_{r}>i_{r+1}=\ldots=i_{k}>0$, or $\mathbf{i}^{\prime}=$ $\left(i_{1}+1\right)$, if $i_{1}=\ldots=i_{k}>0$. Note that $\mathbf{i}^{\prime}$ is not defined when $\mathbf{i}$ has the form $\mathbf{i}=(n, \ldots, n)$.

If $\mathbf{i}=\left(i_{1}, \ldots, i_{k}\right)$, we denote by $\mu(\mathbf{i})$ the number of Boardman symbols $\mathbf{j}=\left(j_{1}, \ldots, j_{k}\right)$ such that $j_{r} \leq i_{r}$, for $r=1, \ldots, k$ and $j_{1}>0$.

Finally, we define $v(\mathbf{i}, n, p)$ as the number

$$
\left(p-n+i_{1}\right) \mu\left(i_{1}, \ldots, i_{k}\right)-\left(i_{1}-i_{2}\right) \mu\left(i_{2}, \ldots, i_{k}\right)-\ldots-\left(i_{k-1}-i_{k}\right) \mu\left(i_{k}\right),
$$

provided that $\mathbf{i}=\left(i_{1}, \ldots, i_{k}\right)$. It is shown in [1] that $v(\mathbf{i}, n, p)$ is the codimension of the Boardman manifold $\Sigma^{\mathbf{i}}$ in the jet space $J^{k}(n, p)$. To simplify the notation, when the dimensions $n, p$ are clear from the context, we shall use $v(\mathbf{i})$ instead of $v(\mathbf{i}, n, p)$ (note that this number depends only on the difference $p-n$ ).

In next proposition we summarize some results of [9] that we are going to use.

Proposition 3.1. We have the following properties for Boardman symbols $\mathbf{i}, \mathbf{j}$ and a map germ $f: K^{\prime \prime}, 0 \rightarrow K^{\prime \prime}, 0$ :

(i) If $\mathbf{i} \leq \mathbf{j}$, then $J_{\mathbf{i}}(f) \subset J_{\mathbf{j}}(f)$;

(ii) $\Sigma^{\mathbf{i}}(f)=V\left(J_{\mathbf{i}}(f)\right) \backslash V\left(J_{\mathbf{i}^{\prime}}(f)\right)$, where $V(I)$ denotes the set germ of zeros in $\left(K^{\prime \prime}, 0\right)$ of an ideal $I \subset \mathscr{E}_{1 \prime}$. (We are using the convention that $V\left(J_{\mathbf{i}^{\prime}}(f)\right)=\varnothing$ when $\mathbf{i}^{\prime}$ is not defined.)

Corollary 3.2. Let $f ; K^{\prime \prime}, 0 \rightarrow K^{\prime \prime}, 0$ be a map germ. For each Boardman symbol $\mathbf{i}$ we have that $V\left(J_{\mathbf{i}}(f)\right)=\Sigma^{\mathrm{i}}(f) \cup \Sigma^{\mathbf{i}^{\prime}}(f) \cup \ldots \cup \Sigma^{\mathrm{i}^{(\prime \prime}}(f)$, where $\mathbf{i}^{\prime}, \ldots, \mathbf{i}^{(\prime)}$ are the iterated successors of $\mathbf{i}$. Moreover, $c_{\mathbf{i}}(f) \geq 1$ if and only if $f$ is a singularity of type $\Sigma^{\mathbf{i}} \cup \Sigma^{\mathbf{i}^{\prime}} \cup \ldots \cup \Sigma^{\mathbf{i}^{(\prime \prime}}$.

Proof. From the above proposition we deduce that

$$
V\left(J_{\mathbf{i}}(f)\right)=\Sigma^{\mathbf{i}}(f) \cup V\left(J_{\mathbf{i}^{\prime}}(f)\right),
$$

and then the required result follows by applying this recursively. The second part is an obvious consequence of the first one, since $c_{\mathbf{i}}(f) \geq 1$ if and only if the ideal $J_{\mathbf{i}}(f)$ is proper, that is, $0 \in V\left(J_{\mathbf{i}}(f)\right)$.

EXAMPLE 3.3. Note that we have $\overline{\Sigma^{i}(f)} \subseteq V\left(J_{\mathbf{i}}(f)\right)$, where $\overline{\Sigma^{\mathbf{i}}(f)}$ denotes the closure of 
$\Sigma^{\mathrm{i}}(f)$ in the Zariski topology. However, the equality is not true in general. For instance, consider the map germ $f: \mathbb{C}^{5} \rightarrow \mathbb{C}^{5}$ given by

$$
f(u, v, w, x, y)=\left(u, v, w, x y, x^{2}+y^{2}+u x+v y\right) .
$$

It is a singularity of type $\Sigma^{2,0}$ and is $\mathscr{A}$-stable. For $\mathbf{i}=(1,1,1,1,1)$ we have that $\overline{\Sigma^{1.1 .1 .1 .1}(f)}=\Sigma^{1,1,1,1.1}(f)=\varnothing$. But the above corollary gives that

$$
V\left(J_{1,1,1,1,1}(f)\right)=\Sigma^{1,1,1,1,1}(f) \cup \Sigma^{2}(f) \ldots \Sigma^{5}(f) \neq \varnothing .
$$

On the other hand, the above corollary can be improved in some particular cases.

Corollary 3.4. Let $f: K^{n}, 0 \rightarrow K^{p}, 0$ be a map germ and $\mathbf{i}$ a Boardman symbol.

(i) Suppose that $f$ is a singularity of type $\Sigma^{\mathbf{i}}$, then $V\left(J_{\mathbf{i}}(f)\right)=\Sigma^{\mathbf{i}}(f)$. Moreover, if $c_{\mathrm{i}}(f)=1$ we have that $f$ is a singularity of type $\Sigma^{\mathrm{i}, 0}$.

(ii) Suppose that $f$ has rank $n-1$, then $V\left(J_{1, \ldots, 1}(f)\right)=\Sigma^{1, \ldots, 1}(f)$.

Proof. The fact that the rank is an upper semicontinuous function implies that if $f$ is a singularity of type $\Sigma^{i}$, then $\Sigma^{i}(f)=\ldots=\Sigma^{i \prime \prime}(f)=\varnothing$, which gives the first part of (i).

For the second one, suppose that $c_{\mathrm{i}}(f)=1$. Then we have that $J_{\mathrm{i}}(f)=\mathcal{M}_{n}$, being $\mathcal{M}_{n}$ the maximal ideal of the local ring $\mathscr{E}_{n}$. This implies that $g=\left(g_{1}, \ldots, g_{r}\right)$ has rank $n$, where $J_{\mathrm{i}}(f)$ is generated by $g_{1}, \ldots, g_{r}$. Therefore, $(f, g)$ has also rank $n$ and $f$ is a singularity of type $\Sigma^{\mathrm{i}, 0}$.

Finally, the same argument that the rank is an upper semicontinuous function gives that when $f$ has rank $n-1$, then $V\left(J_{1, \ldots, 1}(f)\right)=\Sigma^{1 \ldots . .1}(f)$.

EXAMPLE. The converse of the second part of 1 in the above corollary is not true, even in the case that $f$ is $\mathscr{A}$-stable. For instance, consider the map germ $f(x, y)=\left(x, y^{2}\right)$, which is of type $\Sigma^{1,0}$; however, $c_{1}=\infty$.

Proposition 3.5. Let $f: K^{n}, 0 \rightarrow K^{p}, 0$ be a map germ of type $\Sigma^{i}$ which is generic in the sense of Thom-Boardman, with $v(\mathbf{i})=n$ (and therefore of type $\left.\Sigma^{\mathrm{i}, 0}\right)$. Then $c_{\mathbf{i}}(f)=1$.

Proof. Since $f$ is generic and $v(\mathbf{i})=n, f$ must be a singularity of type $\Sigma^{i, 0}$. Then it follows from the definition of the Boardman symbol that we can select $g_{1}, \ldots, g_{n} \in J_{i}(f)$ with rank $n$ in 0 . But this implies that $J_{\mathbf{i}}(f)=\left\langle g_{1}, \ldots, g_{n}\right\rangle=\mathcal{M}_{n}$, and hence $c_{\mathbf{i}}(f)=1$.

4. Geometrical interpretation. In this section we restrict ourselves to the case $K=\mathbb{C}$. We want to determine when the number $c_{\mathrm{i}}(f)$ can be interpreted geometrically as the number of $\Sigma^{\mathbf{i}}$ points that appear in a generic deformation of $f$. To do this, we first study when the number $c_{\mathrm{i}}(f)$ is finite.

One would expect that when $f$ is finitely determined and the codimension of $\Sigma^{\mathbf{i}}$ is large enough (for instance, $v(\mathbf{i}) \geq n$ ), then $c_{\mathrm{i}}(f)<\infty$. However, this is not true. For instance, consider the map germ $f: \mathbb{C}^{5} \rightarrow \mathbb{C}^{5}$ of Example 3.3. It is a singularity of type $\Sigma^{2,0}$ and is $\mathscr{A}$-stable. On the other hand, the Boardman symbol $\mathbf{i}=(1,1,1,1,1)$ satisfies that $v(\mathbf{i})=5$. But a minor computation using Proposition 2.5 gives that $J_{\mathbf{i}}(f) \subset\langle u, v, x, y\rangle$ and thus $c_{\mathrm{i}}(f)=\infty$.

Lemma 4.1. Let $\mathbf{i}$ be a Boardman symbol such that $v(\mathbf{i}), v\left(\mathbf{i}^{\prime}\right), \ldots, v\left(\mathbf{i}^{(t)}\right) \geq n$, where $\mathbf{i}^{\prime}, \ldots, \mathbf{i}^{(t)}$ are the iterated successors of $\mathbf{i}$. If $f: \mathbb{C}^{n}, 0 \rightarrow \mathbb{C}^{p}, 0$ is a finitely determined map germ, then $c_{\mathrm{i}}(f)<\infty$. 
Proof. We have that $c_{\mathrm{i}}(f)=\operatorname{dim}_{\mathbb{C}}\left(\mathscr{E}_{n} / J_{\mathrm{i}}(f)\right)<\infty$ if and only if the Krull dimension of the ring $\mathscr{E}_{n} / J_{\mathrm{i}}(f)$ is zero. But this dimension coincides with $\operatorname{dim} V\left(J_{\mathrm{i}}(f)\right)$ and by Corollary 3.2 this set can be written as

$$
V\left(J_{\mathrm{i}}(f)\right)=\Sigma^{\mathrm{i}}(f) \cup \Sigma^{\mathrm{i}^{\prime}}(f) \cup \ldots \cup \Sigma^{\mathrm{i}(t)}(f) .
$$

On the other hand, we can use the Mather-Gaffney finite determinacy criterion, which says that there is a representative $f: U \rightarrow \mathbb{C}^{p}$ so that $f$ is stable on $U \backslash\{0\}$ (see [12]). Then $j^{k} f$ is transversal to all the Boardman submanifolds on $U \backslash\{0\}$ and thus $V\left(J_{\mathbf{i}}(f)\right) \cap$ $(U \backslash\{0\})$ is a finite union of submanifolds of codimension $\geq n$. By shrinking the neighbourhood $U$ if necessary, we will have that $V\left(J_{\mathbf{i}}(f)\right) \cap(U \backslash\{0\})=\varnothing$, which means that $V\left(J_{\mathbf{i}}(f)\right) \subset\{0\}$ and $\operatorname{dim} V\left(J_{\mathbf{i}}(f)\right)=0$, as required.

Again this result can be improved in some particular cases. The following lemma can be proved by using the same argument than in Lemma 4.1.

LEMMA 4.2. Let $f: \mathbb{C}^{n}, 0 \rightarrow \mathbb{C}^{p}, 0$ be a finitely determined map germ and $\mathbf{i}$ a Boardman symbol such that $v(\mathbf{i}) \geq \mathrm{n}$. Then $c_{\mathrm{i}}(f)<\infty$ provided that either

(i) $f$ is a singularity of type $\Sigma^{\mathbf{i}}$; or

(ii) f has rank $n-1$ and $\mathbf{i}=(1, \ldots, 1)$.

Before stating the main theorem of this section, we give the following lemma. It is based on a standard argument and shows that the Cohen-Macaulay property in necessary in order to compute the number of $f$ from the number $c_{\mathrm{i}}(f)$.

LEMMA 4.3. Let $f: \mathbb{C}^{n}, 0 \rightarrow \mathbb{C}^{p}, 0$ be a finitely determined map germ and $\mathbf{i}$ a Boardman symbol such that $v(\mathbf{i})=n$ and $v\left(\mathbf{i}^{\prime}\right), \ldots, v\left(\mathbf{i}^{(t)}\right)>n$. Let $F(u, x)=\left(u, f_{u}(x)\right)$ be a 1parameter unfolding of $f$ with the property that $f_{u}$ is generic for $u \neq 0$. Then, the number of $\Sigma^{\mathbf{i}}$ points of $f_{u}$, for $u \neq 0$ is equal to $c_{\mathbf{i}}(f)$ if and only if the local ring $\mathscr{E}_{n+1} / J_{\mathbf{i}}(F)$ is Cohen-Macaulay.

Proof. If $c_{\mathrm{i}}(f)=0$, then $V\left(J_{\mathrm{i}}(f)\right)=\varnothing$ and since $V\left(J_{\mathrm{i}}\left(f_{u}\right)\right)=\Sigma^{\mathbf{i}}\left(f_{u}\right)$ for $u \neq 0, f_{u}$ will not have any $\Sigma^{i}$ point. Therefore, we can suppose that $c_{\mathbf{i}}(f)>0$ and $V\left(J_{\mathbf{i}}(f)\right)=\{0\}$ by the above lemma.

In this case, the set germ $X=V\left(J_{\mathrm{i}}(F)\right)$ is 1-dimensional and the projection $\pi: X \rightarrow \mathbb{C}$ given by $\pi(u, x)=u$ satisfies that $\pi^{-1}(0)=\{0\}$. Moreover, for $u \neq 0$, the cardinal of $\pi^{-1}(u)$ is equal to the number of $\Sigma^{\mathbf{i}}$ points that appear in $f_{t}$. But this number is equal, by the formula of Samuel (see for instance $[\mathbf{1 0}]$ ), to the multiplicity $e(\langle\bar{u}\rangle, R)$, where $R=$ $\mathscr{E}_{n+1} / J_{\mathrm{i}}(F)$ and $\bar{u}$ denotes the class of $u$ in $R$.

On the other hand, since $\langle\bar{u}\rangle$ is a parameter ideal of $R$, we apply Theorem 17.11 of [6] and get that $R$ is Cohen-Macaulay if and only if $e(\langle\bar{u}\rangle, R)=\operatorname{dim}_{\mathbb{C}} R /\langle\bar{u}\rangle$. Finally, note that

$$
\operatorname{dim}_{\mathbb{C}} \frac{R}{\langle\bar{u}\rangle}=\operatorname{dim}_{\mathbb{C}} \frac{\mathscr{E}_{n+1} / J_{\mathbf{i}}(F)}{\langle\bar{u}\rangle}=\operatorname{dim}_{\mathbb{C}} \frac{\mathscr{E}_{n}}{J_{\mathbf{i}}(f)}
$$

THEOREM 4.4. Let $f: \mathbb{C}^{\prime \prime}, 0 \rightarrow \mathbb{C}^{p}, 0$ be a finitely determined map germ and $\mathbf{i} a$ Boardman symbol such that $v(\mathbf{i})=n$. Then $c_{\mathbf{i}}(f)$ is the number of $\Sigma^{\mathbf{i}}$ points that appear in a generic deformation of $f$, provided that either 
(i) the length of $\mathbf{i}$ is 1 ;

(ii) $f$ is a singularity of type $\Sigma^{i}$; or

(iii) $f$ has rank $n-1$ and $\mathbf{i}=(1, \ldots, 1)$.

Proof. Let $F: \mathbb{C}^{n+1}, 0 \rightarrow \mathbb{C}^{p+1}, 0$ be a 1-parameter unfolding of $f$, given by $F(u, x)=\left(u, f_{u}(x)\right)$, and with the property that $f_{u}$ is generic for $u \neq 0$. By the above lemma, we have to show that in the three cases, the ring $R=\mathscr{E}_{n+1} / J_{i}(F)$ is CohenMacaulay.

In the first case, $J_{\mathbf{i}}(F)$ is defined by the $\left(n-i_{1}+1\right)$-minors of a matrix of order $n \times p$, being $\mathbf{i}=i_{1}$. Since $v(\mathbf{i})=i_{1}\left(p-n-i_{1}\right)=n$, we have that $\operatorname{dim} R=1=(n+1)-i_{1}(p-n-$ $\left.i_{1}\right)$, which implies that $R$ is a determinantal ring and therefore is Cohen-Macaulay.

In the second case, $F$ is also a singularity of type $\Sigma^{\mathbf{i}}$ and thus $V\left(J_{\mathbf{i}}(F)\right)=\Sigma^{\mathbf{i}}(F)$. This means that the local ring $R$ can be obtained as the pull back of the local ring of the Boardman submanifold $\Sigma^{i} \subset J^{k}(n, p)$ through the map $j^{k} F: \mathbb{C}^{n+1}, 0 \rightarrow J^{k}(n, p)$. Now, $\Sigma^{i}$ is Cohen-Macaulay because it is smooth at every point and $\operatorname{since} \operatorname{codim} \Sigma^{i}=n=\operatorname{codim} R, R$ is also Cohen-Macaulay.

In the last case, we have that $F$ has rank $n$. By Corollary 2.6 we know that after a coordinate change in the source, the ideal $J_{i}(F)$ is generated by $n$ functions $g_{1}, \ldots, g_{n}$. But $R=\mathscr{E}_{n+1} / J_{\mathrm{i}}(F)$ has dimension one and thus it is a complete intersection. In particular, it is Cohen-Macaulay (see [6] for instance, for the definitions and properties used here).

Note that the first case of the above theorem includes the Milnor number for $p=1$ and the number of cross caps for $n=2$ and $p=3$. More generally, we have that $c_{1}(f)$ is the number of $\Sigma^{1}$ points of a finitely determined map germ $f: \mathbb{C}^{n}, 0 \rightarrow \mathbb{C}^{2 n-1}, 0, c_{2}(f)$ is the number of $\Sigma^{2}$ points of $f: \mathbb{C}^{2 n}, 0 \rightarrow \mathbb{C}^{3 n-2}, 0$, etc.

On the other hand, if we consider the general case of a finitely determined map germ $f: \mathbb{C}^{n}, 0 \rightarrow \mathbb{C}^{p}, 0$ and a Boardman symbol $\mathbf{i}$ with $v(\mathbf{i})=n$, we can try to apply the above argument to prove that $c_{\mathbf{i}}(f)$ is the number of $\Sigma^{\mathbf{i}}$ points. After Corollary 3.2 and Lemma 4.1 it is obvious that we must add the condition that $v\left(\mathbf{i}^{\prime}\right), \ldots, v\left(\mathbf{i}^{(t)}\right)>n$ in order to ensure that $c_{\mathbf{i}}(f)$ is finite and that $f_{u}$ has only $\Sigma^{\mathbf{i}}$ points as isolated singularities. However, even in this case the result is not true in general. In fact, the local ring $R=\mathscr{E}_{n+1} / J_{\mathrm{i}}(F)$ that appears in the above proof is not Cohen-Macaulay in general and this is due to the fact that these rings do not have a reduced structure (it is well known that every one dimensional reduced local ring is Cohen-Macaulay). The following example will illustrate this with more detail.

Example 4.5. When $n=p=3$ the only Boardman symbol that satisfies $v(\mathbf{i})=3$ is $\mathbf{i}=(1,1,1)$. Moreover, its iterated successors are $\mathbf{i}^{\prime}=2$, with $v\left(\mathbf{i}^{\prime}\right)=4$ and $\mathbf{i}^{\prime \prime}=3$, with $v\left(\mathbf{i}^{\prime \prime}\right)=9$.

Let $f: \mathbb{C}^{3}, 0 \rightarrow \mathbb{C}^{3}, 0$ be the map germ given by $f(x, y, z)=\left(x, y z, y^{2}+z^{2}+x z\right)$. We will show that for this map germ the number $c_{1,1,1}(f)$ is 4 , but the 1-parameter generic deformation $f_{u}(x, y, z)=\left(x, y z, y^{2}+z^{2}+x z+u y\right)$ just has two $\Sigma^{1,1,1}$ points for $u \neq 0$.

The first step is to compute the ideal $J_{1,1,1}(F)$. It is generated by the maximal minors of the matrix

$$
\left(\begin{array}{rrrrr}
z & 2 y+u & -4 y-u & 8 y+u & 16 z+6 x \\
y & 2 z+x & 4 z+x & 8 z+x & 16 y+6 u
\end{array}\right)
$$


In the case $u=0$, it is easy to see that $J_{1,1,1}(f)=\mathcal{M}_{3}^{2}$, where $\mathcal{M}_{3}=\langle x, y, z\rangle$, the maximal ideal of $\mathscr{E}_{3}$. Therefore $c_{1,1,1}(f)=4$.

On the other hand, we have $V\left(J_{1,1,1}(F)\right)=V\left(4 z+x, 4 y+u, x^{2}-u^{2}\right)$, so that for $u \neq 0$, the $\Sigma^{1,1,1}$ points of $f_{u}$ are

$$
P_{1}=\left(u,-\frac{u}{4},-\frac{u}{4}\right), \quad P_{2}=\left(-u,-\frac{u}{4}, \frac{u}{4}\right)
$$

Finally, we see that $f_{u}$ is in fact generic for $u \neq 0$, by showing that $P_{1}$ and $P_{2}$ are $\Sigma^{1,1,1,0}$ points. We must prove that the rank of $f_{11}$ and the generators of $J_{1,1,1}\left(f_{t \prime}\right)$ is equal to 3 at both points. We consider the minor given by the first and the last columns, which is equal to $-6 x y+6 u z$. Then the jacobian determinant of $(x, x y,-6 x y+6 u z)$ gives

$$
6 x y+6 u z
$$

which is equal to $-3 u^{2}$ at $P_{1}$ and $3 u^{2}$ at $P_{2}$. This shows that the only singularities that appear in $f_{u \prime}$ are $\Sigma^{1.0}, \Sigma^{1.1 .0}$ or $\Sigma^{1.1,1.0}$. Then we can use the canonical forms of Morin [8] and deduce that $f_{u}$ is generic at every point.

5. Singularities of projections Let $g: K^{N}, 0 \rightarrow K^{p}, 0$ be a submersive map germ, so that $g^{-1}(0)$ is a submanifold germ of codimension $p$ of $K^{N}$. Suppose that $K^{N}=K^{n} \times K^{q}$ and let $\pi: K^{N}, 0 \rightarrow K^{n}, 0$ be the projection given by $\pi(x, y)=x$. Our purpose is to determine the number $c_{i}\left(\left.\pi\right|_{g^{-1}(0)}\right)$ in terms of the partial derivatives of $g$ with respect to the coordinates $y_{j}$.

THEOREM 5.1. Let $g: K^{N}, 0 \rightarrow K^{p}, 0$ be a submersive map germ and let $\pi: K^{N}, 0 \rightarrow K^{n}$, 0 be the projection as above. Suppose that $\left.\pi\right|_{g^{-1}(0)}$ has rank $r$. Then

$$
c_{\mathbf{i}}\left(\left.\pi\right|_{g^{-1}(0)}\right)=\operatorname{dim}_{K} \frac{\mathscr{E}_{N}}{I_{g}+J_{\mathbf{j}}(g ; y)},
$$

where $I_{s}=\left\langle g_{1}, \ldots, g_{p}\right\rangle$ and

$$
\mathbf{j}= \begin{cases}\mathbf{i}, & \text { when } n-p<r \\ \mathbf{i}-(n-p-r)(1, \ldots, 1), & \text { when } n-p \geq r\end{cases}
$$

We start by showing that the ideal $I_{g}+J_{\mathbf{j}}(g ; y)$ that appears in the above theorem, does not depend on the map germ $g$, but only depends on the submanifold $g^{-1}(0)$.

LEMma 5.2. Suppose that $f$ and $g: K^{N}, 0 \rightarrow K^{p}, 0$ are two submersive map germs such that $f^{-1}(0)=g^{-1}(0)$. Then

$$
I_{g}+J_{\mathbf{j}}(g ; y)=I_{f}+J_{\mathbf{j}}(f ; y) .
$$

Proof. We parameterize the submaifold $g^{-1}(0)=f^{-1}(0)$ by an immersion $\varphi: K^{N-p}$, $0 \rightarrow K^{N}, 0$, which induces an epimorphism $\varphi^{*}: \mathscr{E}_{N} \rightarrow \mathscr{E}_{N-p}$. Then, by using the local form of an immersion/submersion, it is not very difficult to show that $I_{f}=I_{g}=\operatorname{ker} \varphi^{*}$.

Now, suppose that $\mathbf{j}=\left(j_{1}, \ldots, j_{k}\right)$. We prove by induction on $k$ the required condition. For $k=1$ we have

$$
I_{g}+J_{j_{1}}(g ; y)=I_{g}+\Delta_{n-j_{1}+1}(g,\{0\} ; y)=\Delta_{n-j_{1}+1}\left(I_{g} ; y\right),
$$


where the last equality comes from property 4 of Lemma 2.2. Since the same can be stated for $f$, the result is a consequence of $I_{f}=I_{g}$.

Finally, a similar argument can be used to prove that if the result is true for $k-1$, then it is also true for $k$, which concludes the proof of the lemma.

Suppose now that the map germ $\left.\pi\right|_{g^{-1}(0)}$ has rank $r$ and let $s=N-p-r$. We must distinguish the two cases: $n-p<r$ or $n-p \geq r$.

1. Case $n-p<r$. In order to simplify the notation we rewrite the coordinates of $K^{N}$ as $(z, u, v, w)$, being $z \in K^{r}, u \in K^{n-r}, v \in K^{s}$ and $w \in K^{q-s}$. With this notation, we can parameterize the submanifold $g^{-1}(0)$ by an immersion $\varphi: K^{N-p}, 0 \rightarrow K^{N}, 0$ of the form $\varphi(z, v)=(z, \psi(z, v), v, \eta(z, v))$, for some map germs $\psi: K^{N-p}, 0 \rightarrow K^{n-r}, 0$ and $\eta: K^{N-p}$, $0 \rightarrow K^{q-s}, 0$.

Then we can apply the above lemma and suppose that $g$ is defined by

$$
g_{i}(z, u, v, w)= \begin{cases}\psi_{i}(z, v)-u_{i}, & \text { for } i=1, \ldots, n-r \\ \eta_{i-(n-r)}(z, v)-w_{i-(n-r),}, & \text { for } i=n-r+1, \ldots, p .\end{cases}
$$

On the other hand, $\left.\pi\right|_{g^{-1}(0)}$ is $\mathscr{A}$-equivalent to the map germ $\pi \circ \varphi$ given by

$$
\pi \circ \varphi(z, v)=(z, \psi(z, v)) \text {. }
$$

2, Case $n-p \geq r$. This case is simpler than the above. Now we have $q<s$ and thus we only need to consider $(z, u, v)$, with $z \in K^{r}, u \in K^{n-r}$ and $v \in K^{s}$, as coordinates of $K^{N}$.

The parameterization of $g^{-1}(0)$ is now given by the immersion $\varphi(z, v)=$ $(z, \psi(z, v), v)$, and thus we can suppose that $g$ is defined by

$$
g_{i}(z, u, v)=\psi_{i}(z, v)-u_{i}, \quad \forall i=1, \ldots, n-r .
$$

Finally, the projection $\pi \circ \varphi$ has the same expression than above:

$$
\pi \circ \varphi(z, v)=(z, \psi(z, v)) \text {. }
$$

Proof of Theorem 5.1. By Proposition 2.5, we have that

$$
c_{\mathbf{i}}(\pi \circ \varphi)=c_{\mathbf{i}}(\psi ; v)=\operatorname{dim}_{K} \frac{\mathscr{E}_{N-p}}{J_{\mathbf{i}}(\psi ; n)} .
$$

But the immersion $\varphi$ induces a ring epimorphism $\varphi^{*}: \mathscr{E}_{N} \rightarrow \mathscr{C}_{N-p}$, whose kernel is given by the ideal $I_{g}$. Then we have an isomorphism

$$
\varphi^{*}: \frac{\mathscr{E}_{N}}{I_{g}} \rightarrow \mathscr{E}_{N-p}
$$

Now, suppose that $\mathbf{i}=\left(i_{1}, \ldots, i_{k}\right)$. We prove by induction on $k$ that $\varphi^{*}\left(J_{\mathbf{j}}(g ; v, w)\right)=$ $J_{\mathbf{i}}(\psi ; v)$ and thus we have the required result.

In the case $n-p<r$, the jacobian matrix of $g$ with respect to the coordinates $v, w$ has the form $\left(\begin{array}{cc}A & 0 \\ B & -I_{q-s}\end{array}\right)$, where $A=\left(\partial \psi_{i} / \partial v_{j}\right)$ is the jacobian matrix of $\psi$ with respect the coordinates $v_{j}, B=\left(\partial \eta_{i} / \partial v_{j}\right)$ is the jacobian matrix of $\eta$ with respect the coordinates $v_{j}$ and $I_{q-s}$ is the identity matrix of order $q-s$. The ideal generated by the minors of order $s-i_{1}+1$ of $A$ is the same than the ideal generated by the minors of order 
$q-i_{1}+1$ of the whole matrix. Thus the above assertion is clear for $k=1$, taking $\mathbf{j}=\mathbf{i}$. A similar argument shows that if the assertion is true for $k-1$, then it is also true for $k$.

In the other case, $n-p \geq r$, the jacobian matrix of $g$ with respect to the coordinates $v$ is just the top row of the above matrix. Then $A$ and the whole matrix have the same minors of order $s-i_{1}+1$. Therefore, we must adjust the size by taking $j_{1}=i_{1}-(s-q)=$ $i_{1}-(n-p-r)$, so that the assertion is true again.

We conclude the paper with some applications of Theorem 5.1. For instance, if we consider the particular case $q=p=1$, we have a submersive function germ. $g: K^{n+1}$, $0 \rightarrow K, 0$. Given any projection $\pi: K^{n+1}, 0 \rightarrow K^{n}, 0$, the restriction $\left.\pi\right|_{g^{-1}(0)}$ will have rank at least $n$. Then we know from Corollary 2.6 that the only Boardman symbols $\mathbf{i}$ that give non trivial numbers $c_{\mathbf{i}}\left(\left.\pi\right|_{g^{-1}(0)}\right)$, are those of the form $\mathbf{i}=(1, \ldots, 1)$.

Corollary 5.3. Let $g: K^{n+1}, 0 \rightarrow K, 0$ be a submersive function germ and let $\pi: K^{n+1}$, $0 \rightarrow K^{n}, 0$ be the projection given by $\pi\left(x_{1}, \ldots, x_{n}, t\right)=\left(x_{1}, \ldots, x_{n}\right)$. Then

$$
c_{1, k \text { timu, }, 1}\left(\left.\pi\right|_{g^{-1}(0)}\right)=\operatorname{dim}_{K} \frac{\mathscr{E}_{n+1}}{\left\langle g, \frac{\partial g}{\partial t}, \ldots, \frac{\partial^{k} g}{\partial t^{k}}\right\rangle} .
$$

Other application of Theorem 5.1 can be observed for catastrophe maps. Suppose that we have a potential function $F: K^{r} \times K^{\prime \prime} \rightarrow K$ given by $F(u, x)=F_{u}(x)$. Then the catastrophe manifold is defined as the set

$$
M_{F}=\left\{(u, x) \in K^{r} \times K^{n}: \frac{\partial F}{\partial x_{i}}(u, x)=0, \forall i=1, \ldots, n\right\}=(\nabla F)^{-1}(0),
$$

where $\nabla F(u, x)=\nabla F_{u}(x)$ denotes the gradient vector of the potential function $F_{u}$ with respect to the variables $x_{i}$. Now, the catastrophe map $\chi_{F}: M_{F} \rightarrow K^{r}$ is just the restriction of the projection $\pi: K^{r} \times K^{n} \rightarrow K^{r}$ given by $\pi(u, x)=u$ (see [11]).

Corollary 5.4. Let $F: K^{r} \times K^{\prime \prime} \rightarrow K$ be a potential function germ such that the gradient vector $\nabla F: K^{r} \times K^{\prime \prime} \rightarrow K^{\prime \prime}$ is a submersion. Suppose that $\chi_{F}$ has rank $l$. Then at each point of $M_{F}$ we have

$$
c_{\mathrm{i}}\left(\chi_{r}\right)=\operatorname{dim}_{\kappa} \frac{\mathscr{E}_{r+n}}{I_{\nabla F}+J_{\mathbf{j}}(\nabla F ; x)},
$$

where $I_{\Gamma F}=\left\langle\frac{\partial F}{\partial x_{1}}, \ldots, \frac{\partial F}{\partial x_{n}}\right\rangle$ and

$$
\mathbf{j}= \begin{cases}\mathbf{i}, & \text { when } r-n<l \\ \mathbf{i}-(r-n-l)(1, \ldots, 1), & \text { when } r-n \geq l\end{cases}
$$

\section{REFERENCES}

1. J. Boardman, Singularities of differentiable maps, Inst. Hautes Études Sci. Publ. Math. 33 (1967), $21-57$.

2. T. Fukuda and G. Ishikawa, On the number of cusps of stable perturbations of a plane-to-plane singularity, Tokyo J. Math., 10 (1987), 375-384. 
3. W. Fulton, Intersection theory (Springer-Verlag, 1984).

4. T. Guffney and D. Mond, Cusps and double folds of germs of analytic maps $\mathbb{C}^{2} \rightarrow \mathbb{C}^{2}, J$. London Math. Soc. 243 (1991), 185-192.

5. J. N. Mather, Stable map-germs and algebraic geometry, in Manifolds-Amsterdam, Lect. Notes in Math. 197 (Springer-Verlag, 1971) 176-193.

6. H. Matsumura, Commutative ring theory (Cambridge Studies in Advanced Math. 8, Cambridge University Press, 1986).

7. D. Mond, Vanishing cycles for analytic maps, in Singularity Theory and its Applications, Lecture Notes in Math. 1462 (Springer-Verlag, 1991) 221-234.

8. B. Morin, Formes canoniques des singularités d'une application différentiable, C. R. Acad. Sci. Paris 260 (1965), 5662-5665, 6503-6506.

9. B. Morin, Calcul jacobien, Ann. Sci. École Norm. Sup. 8 (1975), 1-98.

10. D. Mumford, Algebraic Geometry I Complex Projective Varieties, A series of Comprehensive Studies in Mathematics 221 (Springer-Verlag, Berlin, Heidelberg, 1976).

11. D. J. A. Trotman and E. C. Zeeman, The classification of elementary catastrophes of codimension $\leq 5$, in Structural Stability, the Theory of Catastrophes and Applications in the Sciences, Seattle 1975, Lecture Notes in Math. 525 (Springer-Verlag, 1976).

12. C. T. C. Wall, Finite determinacy of smooth map-germs, Bull. London Math. Soc. 13 (1981), 481-539.

\author{
J. J. Nuño Ballesteros \\ Departament de Geometria I Topologia, Universitat de València \\ Campus de Burjassot, 46100 Burjassot, SPAIN \\ email: nuno@uv.es
}

M. J. SAIA

Instituto de Geociênclas e Ciẽncias Exatas, Universidade Estadual Paulista

Campus de Rio Claro, Caixa Postal 178, 13500-230, Rio Claro, SP, BRAZIL

email: mjsaia@rcb000.uesp.ansp.br 